\title{
Resultados en cirugía mayor ambulatoria en otorrinolaringología realizada en un hospital de baja complejidad
}

\section{Results in major ambulatory surgery in otorhinolaryngology performed in a hospital of low complexity}

\author{
Daniel Moyano M¹, Rodrigo González E¹, Eduardo Peirano 01, Jaime Bermeo S ${ }^{2,3}$, Carlos Narváez C2,4,
} Arturo Samith $\mathbf{M}^{5}$.

\section{RESUMEN}

Introducción: La cirugía mayor ambulatoria confiere al sistema sanitario un modelo de gestión eficiente y de alta calidad.

Objetivo: Conocer los resultados de la cirugía mayor ambulatoria en otorrinolaringología (ORL) en un hospital de baja complejidad.

Material y método: Estudio descriptivo retrospectivo realizado en el Hospital Santo Tomás de Limache entre los años 2004 y 2009. La inclusión de pacientes sometidos a cirugía otorrinolaringológica bajo la modalidad de cirugía mayor ambulatoria. Se realizó análisis descriptivo en base a promedios, desviación estándar y proporciones. En análisis bivariado, se emplearon las pruebas de chi cuadrado y t-student.

Resultados: Se incorporó 815 pacientes. El 58\% fue de sexo masculino, con edad promedio de 8,4 $\pm 6,2$ años. La adenoamigdalectomía sola o asociada a otras cirugías se efectuó en $54,2 \%$ de los casos. La tasa de complicaciones posoperatorias fue de 1,22\%, la mitad de ella, la hemorragia. No hubo mortalidad. No se encontró asociación estadística entre la presencia de complicaciones y alguna variable en particular.

Conclusiones: En pacientes de otorrinolaringología seleccionados, la cirugía mayor ambulatoria efectuada en un hospital de baja complejidad tiene buenos resultados, con una baja tasa de complicaciones.

Palabras clave: Cirugía mayor ambulatoria, hospital de baja complejidad, otorrinolaringología, complicaciones.

\section{ABSTRACT}

Introduction: Major ambulatory surgery provide to public health and efficient and high quality model.

Aim: To know the results in patient underwent Otorrinolaringologic surgery in a low complexity hospital.

1 Interno de Medicina, Universidad de Valparaíso.

2 Escuela de Medicina, Facultad de Medicina, Universidad de Valparaíso.

3 Servicio de Otorrinolaringología, Hospital Carlos van Buren, Valparaíso.

4 Servicio de Anestesiología, Hospital Carlos van Buren, Valparaíso.

5 Sociedad Chilena de Otorrinolaringología, Medicina y Cabeza y Cuello. 
Material and methods: Retrospective descritive study. Date from 815 who underwent Otorrinolaringologic surgery carried on a major ambulatory surgery basis in Hospital Santo Tomás de Limache, between 2004 and 2009 where retrospectively reviewed. Analysed date included sex, age, type of conducted surgery, type of complications and the period of time in which patients were discharged. Averages, standard deviation and proportions were used. In bivariate analysis, chi square and t-student were used.

Results: $58 \%$ of enrolled patients were males with age average of $8.4 \pm 6.2$ years. Adenotonsillectomy single or associated to other surgeries took place in $54.2 \%$ of the cases. The rate of postoperation side effects was $1.22 \%$, And half of these patient presented postoperatory bleeding. There was no mortality. There was not statistical association between the presence of complications and some variable in particular.

Conclusions: Major ambulatory surgery In selected patients who need otorrinolaringologic surgery in a low complexity hospital is safe with low rate of complication.

Key words: Ambulatory major surgery, hospital of low complexity, otorhinolaryngology, complications.

\section{INTRODUCCION}

La cirugía más frecuente en otorrinolaringología, es la adenoamigdaliana. En nuestro país, se plantea que en un servicio de ORL, el $56,3 \%$ corresponde a cirugías del anillo de Waldeyer ${ }^{1}$ y $27,5 \%$ de las cirugías correspondieron a adenoamigdalectomías ${ }^{1}$.

Actualmente no existe consenso sobre la estadía posoperatoria en esta cirugía, puesto que dependiendo de los centros y de los países, las indicaciones van desde la hospitalización durante una semana al alta inmediata². Este último es un concepto que se ha venido aplicando desde mediados de los años setenta, siendo precursores Maniglia y cols $^{3}$, quienes lo desarrollaron como consecuencia del aumento progresivo de la demanda y del gasto sanitario que conlleva mantener una hospitalización prolongada. Sin embargo, no es sino hasta el año 1986, con la publicación de Davis, que se acuña el término Cirugía Mayor Ambulatoria $(\mathrm{CMA})^{4}$, para referirse al procedimiento sin estadía hospitalaria.

La CMA consiste en un acto quirúrgico programado, realizado en condiciones técnicas de seguridad de un pabellón operatorio, bajo anestesia general y con un periodo de observación variable, sin hospitalización posterior ${ }^{5}$. Son criterios esenciales para efectuar una CMA tener un riesgo hemorrágico mínimo, un bajo riesgo de dolor, parámetros fisiológicos vitales estables, bajo riesgo tromboembólico, duración de la intervención menor de 90 minutos, pacientes ASA I ó II y permanecer a menos de 30 minutos del hospital, y un período de observación de hasta 8 horas posintervención $n^{5,6}$.

La incidencia de complicaciones del procedimiento ambulatorio es en promedio $2 \%$, con valores que van entre $0,7 \%$ y $7 \%{ }^{7,8}$. Las reportadas con mayor frecuencia corresponden a sangrado de la herida operatoria, broncoespasmo, hiperemesis y fiebre ${ }^{7,9,10}$. Dado que éstas ocurren generalmente en las primeras 6 horas del posoperatorio ${ }^{8}$ se recomienda dar de alta después de este período de tiempo ${ }^{11}$.

Al desarrollar la CMA, se logra establecer un modelo de gestión eficiente que facilita el acceso del usuario al sistema y su posterior resolución quirúrgica ${ }^{4}$, ofreciendo una prestación de alta calidad con una relación costo/beneficio más favorable. Por otro lado, en el paciente disminuye la ansiedad que le genera la cirugía, y le permite una rápida incorporación a sus actividades cotidianas. Para el sistema sanitario resulta beneficioso ya que libera camas, reduce los costos y agiliza la asistencia médica. Por último, garantiza al cirujano la calidad del pre y posoperatorio, además de reducir las infecciones nosocomiales ${ }^{7,8}$.

Ya que la mayoría de las experiencias de CMA han sido descritas en grandes centros-2-4,12,13, en este trabajo se pretende evaluar los resultados de esta modalidad de enfoque terapéutico de cirugía otorrinolaringológica en un hospital de baja complejidad. 


\section{OBJETIVO}

Conocer los resultados de la cirugía mayor ambulatoria en otorrinolaringología en un hospital de baja complejidad.

\section{MATERIAL Y METODO}

\section{Diseño}

Estudio descriptivo retrospectivo realizado con los pacientes sometidos a cirugías de la especialidad de ORL en el Hospital Santo Tomás de Limache entre junio de 2004 y diciembre de 2006, y entre julio de 2008 y julio de 2009.

\section{Pacientes}

Se revisaron las fichas de todos los pacientes que cumpliesen los siguientes criterios de inclusión: mayores de 2 años con cirugías de ORL menores a 60 minutos de duración, aprobado y firmado el consentimiento informado (o el adulto responsable en el paciente menor de edad), ASA I ó II, valoración preoperatoria por equipo multidisciplinario (anestesista, otorrinolaringólogo, enfermero y asistente social en casos específicos). Y residencia próxima al hospital, o con transporte garantizado en caso de ser necesario. Además, que tuvieran un entorno familiar adecuado y un nivel sociocultural que brindara las condiciones adecuadas para una buena recuperación.

\section{TÉCNICA QUIRÚRGICA Y MANEJO POSOPERATORIO}

En el acto operatorio se utilizó el electrobisturí en el modo de coagulador, tanto durante la disección como en la hemostasia, cubriendo posteriormente el lecho con subgalato de bismuto. Se utilizó anestesia general con sevofluorano y se utilizó dexametasona por vía endovenosa durante el perioperatorio, como profilaxis de náusea y vómito. No se utilizó óxido nitroso como gas coadyuvante. La analgesia se planificó en base a ketoprofeno y tramadol ${ }^{14}$.
Posterior a la cirugía los pacientes fueron controlados en sala de recuperación posoperatoria por un período de una hora con monitorización de signos vitales, control por anestesista, cirujano y personal paramédico. En el caso de los niños, se permitió la compañía de un familiar. Si la evolución fue favorable, se dejaron en observación en sala común por un tiempo promedio de seis horas. El alta fue dada por el médico tratante si el paciente estaba consciente y orientado, con signos vitales normales, sin dolor 0 de baja intensidad, sin sangrado de la región operatoria, con vía aérea permeable y sin signos de obstrucción, vía oral reestablecida y movilidad conservada.

Al momento del alta, se educó a los pacientes 0 a sus cuidadores sobre la evolución de la recuperación y cómo actuar ante las posibles complicaciones, además de los cuidados básicos a seguir, relativos a la dieta, medicamentos y motivos de consulta precoz. Para asegurar el correcto cumplimiento de las indicaciones los fármacos fueron adquiridos en el hospital y se les dio la facilidad de efectuar consultas telefónicas en caso de surgir dudas. Finalmente, ellos fueron citados a control en siete días para control posquirúrgico y evaluación del alta definitiva.

\section{Mediciones}

Los datos fueron obtenidos desde las bases respectivas y los libros de registros, desarrolladas por el servicio quirúrgico de ORL, además de las fichas clínicas de los pacientes. En este trabajo las cirugías incluidas en el grupo de mayor ambulatoria fueron: amigdalectomía, adenoidectomía, adenoamigdalectomía y sus asociaciones con turbinectomía, punción transtimpánica, uvuloplastía y resección del frenillo sublingual (Tabla 1).

Desde las fuentes ya señaladas se extrajo en cada caso información relativa al sexo y edad del paciente, tipo de cirugía practicada, presencia y tipo de complicaciones y momento del alta respecto a la cirugía.

\section{Análisis estadístico}

Para el análisis de datos se utilizó la planilla de cálculo de Microsoft Excel 2000, y el Predictive 
Tabla 1. Intervenciones incluidas en modalidad de CMA. Hospital Santo Tomás de Limache efectuadas entre junio 2004 y junio 2006, y enero y julio 2009

\begin{tabular}{|lll|}
\hline Intervencion & Frecuencia & Porcentaje \\
\hline Adenoidectomía & 32 & 3,9 \\
Adenoidectomía + PTT* & 23 & 2,8 \\
Adenoidectomía + turbinectomía & 9 & 1,1 \\
Adenoidectomía + uvuloplastía & 1 & 0,1 \\
Adenoidectomía + PTT + turbinectomía & 2 & 0,2 \\
Amigdalectomía & 259 & 31,7 \\
Adenoamigdalectomía & 338 & 41,7 \\
Adenoamigdalectomía + PTT & 82 & 10 \\
Amigdalectomía + PTT & 14 & 1,7 \\
Amigdalectomía + turbinectomía & 30 & 3,7 \\
Adenoamigdalectomía + turbinectomía & 21 & 2,6 \\
Amigdalectomía + uvuloplastía & 2 & 0,2 \\
Amigdalectomía + frenillo sublingual & 2 & 0,2 \\
Adenoamigdalectomía + frenillo & 1 & 0,1 \\
\hline
\end{tabular}

*PTT: punción transtimpánica.

Analytics Software Statistics PASW ${ }^{\circledR}$, versión 18.0.0. (IBM, New York, USA). Para el análisis descriptivo de los datos se utilizaron promedios, desviación estándar y proporciones. En análisis bivariado, se utilizaron las pruebas de chi cuadrado y t de student. En ambos casos, se consideró estadísticamente significativo un $p<0,05$.

\section{RESULTADOS}

Ochocientos quince intervenciones quirúrgicas cumplieron con los criterios definidos de inclusión. Las características generales de los pacientes incluidos y de las cirugías efectuadas se muestran en la Tabla 2. Existió un leve predominio del sexo masculino, con $58 \%$ del total de casos (472/815). La edad promedio fue de $8,4 \pm 6,2$, en un rango que va desde el año hasta los 45 años de edad. La adenoamigalectomía sola 0 asociada a otras cirugías fue la intervención más prevalente, con $54,2 \%$ del total (442/815). Noventa y nueve coma dos por ciento de los pacientes fueron dados de alta el mismo día (809/815). En nuestro grupo, la tasa de complicaciones posoperatorias fue de 1,2\% (10/815).

En relación a las complicaciones, la mitad de ellas fueron hemorragia (5/10), representando $0,6 \%$ del total de las intervenciones incluidas. Los otros 5 casos presentaron complicaciones diversas (fiebre, dolor, hiperemesis y edema agudo de pulmón de presión negativa), todas de curso benigno y autolimitado. No hubo mortalidad. No se encontró asociación estadística entre la presencia de complicación y el tipo de intervención realizada, tal como se muestra en la Tabla 3. Tampoco se asoció algún tipo particular de complicación a algún tipo de cirugía ( $p=0,21)$, edad y complicación $(p=0,44)$ ni entre edad y tipo de cirugía $(p=0,31)$.

\section{DISCUSIÓN Y CONCLUSIONES}

Nuestra población presenta características similares a las reportadas por otros autores, tanto en trabajos extranjeros como nacionales ${ }^{3,8-10}$, tratándose de una población preferentemente pediátrica a quienes se les efectuó una adenoamigdalectomía sola 0 asociada a otros procedimientos quirúrgicos. Lo anterior es esperable al considerar que el proceso fisiopatológico subyacente consiste en la proliferación linfoide del anillo de Waldeyer, manifestada en la hipertrofia de adenoides y amígdalas en conjunto, lo que ocurre con mayor frecuencia en este grupo etario ${ }^{15}$. 
Tabla 2. Características de la población e intervenciones

\begin{tabular}{|lll|}
\hline Sexo & $\mathrm{n}$ & $(\%)$ \\
Masculino & & $(58)$ \\
Femenino & 472 & $(42)$ \\
Edad (años) promedio $\pm \mathrm{DE}$ & 343 & \\
Tipo de cirugía & $8,4 \pm 6,2$ & \\
$\quad$ Adenoidectomía sola & & $(3,9)$ \\
Amigdalectomía sola & 32 & $(31,9)$ \\
Adenoamigdalectomía sola & 259 & $(41,4)$ \\
Adenoidectomía asociada & 338 & $(4,3)$ \\
Amigdalectomía asociada & 35 & $(5,9)$ \\
Adenoamigdalectomía asociada & 48 & $(12,6)$ \\
Complicaciones & 103 & $(1,2)$ \\
Si & & $(98,8)$ \\
No & 10 & $(99,1)$ \\
Alta & 805 & $(0,9)$ \\
Mismo día & & \\
Posteriormente & 808 & 7 \\
\hline
\end{tabular}

Tabla 3. Complicación por tipo de intervención (*)

\begin{tabular}{|lllll|}
\hline Complicacion & Amigdalectomia & Adenoidectomia & Adenoamigdalectomia & Total \\
\hline No & 66 & 300 & 439 & 805 \\
Sí & 1 & 6 & 3 & 10 \\
Total & 67 & 306 & 442 & 815 \\
\hline
\end{tabular}

$\left({ }^{*}\right) p=0,28$.

A nivel internacional se ha publicado una tasa de complicación entre 0,6\% y $7 \%$. Un estudio en el año 1992, en Chile, mostró una tasa de complicaciones de 2,2\% ${ }^{9}$. En nuestro trabajo encontramos una tasa del $1,22 \%$, lo que creemos se sustenta en una buena selección de los pacientes, en contar con un equipo médico y de enfermería con experiencia en el procedimiento y en sus cuidados posoperatorios, además de la educación entregada al paciente, sobre el manejo domiciliario posterior al alta y consulta precoz en caso necesario. Al contar con estos recursos e intervenciones, los riesgos se minimizan al máximo, lo que finalmente se traduce en los resultados obtenidos.
Ninguna complicación se asoció a alguna situación particular. La escasa presencia de casos de dolor severo muy probablemente se deba a que éste se manejó efectiva y precozmente en forma ambulatoria mediante contacto telefónico con un enfermero a cargo del programa de CMA, quien instruyó al paciente 0 a su familiar de modificar la dosis o la frecuencia de uso de la analgesia.

Conforme a lo anterior, podemos afirmar que el desarrollo de la CMA con una baja tasa de complicaciones en un hospital de baja complejidad, es un hecho posible. Surge como un esquema alternativo, cada vez más competitivo a la hospitalización 
convencional, sobre todo en la medida en que se objetivan buenos resultados en calidad y eficiencia. Los beneficios antes señalados la hacen muy recomendable, ya que no aumenta los riesgos para los pacientes y genera una mejor relación costo/beneficio tanto para el hospital como para el equipo y los usuarios del sistema.

\section{Agradecimientos}

Debemos destacar que en la realización de este trabajo fue fundamental el apoyo y colaboración del enfermero universitario Osvaldo Flores y su personal, a cargo de pabellón, quienes en todo momento trabajaron con gran entusiasmo y deseos de concretar esta investigación.

\section{BIBLIOGRAFIA}

1. Ulloa P, Urra A, Valenzuela A, Benavente J. Análisis de la atención intrahospitalaria del Servicio de Otorrinolaringología, Hospital Guillermo Grant Benavente de Concepción, 1993-1997. Rev Otorrinolaringología Cir Cabeza Cuello 1999; 53 : 93-100.

2. M Armengot. CMA en Otorrinolaringología: problemática actual. VI Congreso Nacional De Cirugia Mayor Ambulatoria. Mesa redonda de especialidades quirúrgicas. 21 de Noviembre 2003, Valencia, España.

3. ManigliaAJ, Kushner H, Cozzi L. Adenotonsillectomy. A safe outpatient procedure. Arch Otolaryngol Head Neck Surg 1989; 115: 92-4.

4. Davis JE. History of major ambulatory surgery. In Davis JE (ed). Major Ambulatory Surgery. Baltimor: Williams \& Wilkins 1986; 3-46.
5. Sмітн I. The preoperative period and selection criteria in outpatient surgery. Mapfre Medicina 1997; 8 (supl II): 25-29.

6. Phillips PH, Frost E. Preanesthetic assessment of the ambulatory surgery patient. En Frost, ed. Anesthesiology Clinics of North America. Filadelfia Saunders Company 1990; 8: 801-9.

7. García-Roco, Pérez 0 , Quintana $P$,Correa M, Quintana M. Cirugía mayor ambulatoria de glándulas salivales. Archivo Médico de Camagüey; [serie en Internet] 2004; 8(6).

8. Domínguez L. Estructura y funcionamiento de un servicio de cirugía mayor ambulatoria y de corta hospitalización. Rev Cubana Cir 1999; 38(1): 46-51.

9. Brunetto B, Castilla A, Sacaluga F. La adenoamigdalectomía como un procedimiento ambulatorio. Rev Otorrinolaringol Cir Cabeza Cuello 1992; 52: 167-171.

10. Moreno R, Díaz A, Figueroa A. Cirugía mayor ambulatoria en otorrinolaringología. Rev Cubana Cir 2000; 39(1): 10-6.

11. Wiikmann V, Passarelli F, Caniello M, Di Francesco R, Miziara I. Complicações pós-operatórias em tonsilectomias. Rev Bras Otorrinolaringología 2004; 70 (4): 446-51.

12. Pérez G, Sandó B, Peña E. Cirugía mayor ambulatoria en otorrinolaringología: experiencia de 6 años. Rev Cubana Cir [online] 2008; 47(3): pp. 0-0. Disponible en: www.scielo.cl [Consultado el 10 de junio de 2010].

13. Martínez R. Anestesia ambulatoria en otorrinolaringología. Rev de Anestesiología de México 2005; 28: 143-5.

14. Vergnes F. Analgésie après amygdalectomie. Annales Françaises d'Anesthésie et de Réanimation 2008; 27: 30-6.

15. López G Maite. Adenoides y amígdalas. Rev Chil Pediatr 2001; 72 (3): 251-5. 